OPEN ACCESS

Edited by:

Rafael Maldonado,

Pompeu Fabra University, Spain

Reviewed by:

John F. Honek,

University of Waterloo, Canada

Vincenzo Talesa,

University of Perugia, Italy

*Correspondence:

Naila Rabbani

n.rabbani@qu.edu.qa

Paul J. Thornalley

pthornalley@hbku.edu.qa

Specialty section: This article was submitted to Experimental Pharmacology and Drug Discovery,

a section of the journal

Frontiers in Pharmacology

Received: 20 July 2020 Accepted: 14 September 2020 Published: 09 October 2020

Citation:

Al-Motawa MS, Abbas H, Wijten P, de la Fuente $A, X u e M$, Rabbani $N$ and

Thornalley PJ (2020) Vulnerabilities of the SARS-CoV-2 Virus to

Proteotoxicity-Opportunity for

Repurposed Chemotherapy of COVID-19 Infection.

Front. Pharmacol. 11:585408. doi: 10.3389/fphar.2020.585408

\section{Vulnerabilities of the SARS-CoV-2 Virus to Proteotoxicity-Opportunity for Repurposed Chemotherapy of COVID-19 Infection}

\author{
Maryam S. Al-Motawa ${ }^{1,2}$, Hafsa Abbas ${ }^{3}$, Patrick Wijten ${ }^{2}$, Alberto de la Fuente ${ }^{2}$, \\ Mingzhan $\mathrm{Xue}^{2,3}$, Naila Rabbani ${ }^{4 *}$ and Paul J. Thornalley ${ }^{1,2,3 *}$ \\ ${ }^{1}$ College of Health and Life Sciences, Hamad Bin Khalifa University, Qatar Foundation, Doha, Qatar, ${ }^{2}$ Diabetes Research \\ Center, Qatar Biomedical Research Institute, Hamad Bin Khalifa University, Qatar Foundation, Doha, Qatar, ${ }^{3}$ Clinical \\ Sciences Research Laboratories, Warwick Medical School, University of Warwick, University Hospital, Coventry, United \\ Kingdom, ${ }^{4}$ Department of Basic Medical Science, College of Medicine, QU Health, Qatar University, Doha, Qatar
}

The global pandemic of COVID-19 disease caused by infection with the SARS-CoV-2 coronavirus, has produced an urgent requirement and search for improved treatments while effective vaccines are developed. A strategy for improved drug therapy is to increase levels of endogenous reactive metabolites for selective toxicity to SARS-CoV-2 by preferential damage to the viral proteome. Key reactive metabolites producing major quantitative damage to the proteome in physiological systems are: reactive oxygen species (ROS) and the reactive glycating agent methylglyoxal (MG); cysteine residues and arginine residues are their most susceptible targets, respectively. From sequencedbased prediction of the SARS-CoV-2 proteome, we found 0.8-fold enrichment or depletion of cysteine residues in functional domains of the viral proteome; whereas there was a 4.6-fold enrichment of arginine residues, suggesting SARS-CoV-2 is resistant to oxidative agents and sensitive to MG. For arginine residues of the SARSCoV-2 coronavirus predicted to be in functional domains, we examined which are activated toward modification by $M G$ - residues with predicted or expected low $\mathrm{pK}_{\mathrm{a}}$ by neighboring group in interactions. We found 25 such arginine residues, including 2 in the spike protein and 10 in the nucleoprotein. These sites were partially conserved in related coronaviridae: SARS-COV and MERS. Finally, we identified drugs which increase cellular MG concentration to virucidal levels: antitumor drugs with historical antiviral activity, doxorubicin and paclitaxel. Our findings provide evidence of potential vulnerability of SARS-CoV-2 to inactivation by MG and a scientific rationale for repurposing of doxorubicin and paclitaxel for treatment of COVID-19 disease, providing efficacy and adequate therapeutic index may be established.

Keywords: COVID-19, coronavirus, methylglyoxal, glyoxalase, doxorubicin, paclitaxel, proteomics, bioinformatics 


\section{INTRODUCTION}

A global pandemic of COVID-19 disease caused by infection with the SARS-CoV-2 coronavirus has developed from January 2020. It has produced a global public health emergency with currently $\left(20^{\text {th }}\right.$ July 2020 ) over 14 million infections and $c a$. 600,000 deaths, with both rapidly increasing. New treatments are urgently required for COVID-19 disease until effective vaccines are developed. A rapid route to achieve this is repurposing of existing drugs with previously undisclosed activity against coronavirus infection.

As a strategy to identify drugs for repurposing, we sought to explore whether the SARS-CoV-2 may have vulnerabilities in the viral proteome to modification by endogenous reactive metabolites. Pharmacological increase of reactive metabolites will then produce a virucidal effect and therapeutic response for COVID-19 disease. Important reactive metabolites producing major quantitative modification of the proteome in physiological systems are: reactive oxygen species (ROS) and methylglyoxal (MG) (Winterbourn, 2008; Rabbani and Thornalley, 2015). Key to characterizing the vulnerability of the viral proteome to reactive metabolites, ROS and MG, is location of their susceptible amino acid residue targets in functional domains of viral proteins and activation of these residues toward reaction with reactive metabolites. ROS are formed by mitochondria through trace leakage of electron flux in oxidative phosphorylation, by oxidases and other sources. They are metabolized by antioxidant enzymes, superoxide dismutase, catalase, glutathione peroxidase and peroxiredoxins (Murphy et al., 2011). The reactive dicarbonyl metabolite, MG, is formed mainly by trace level degradation of triosephosphate glycolytic intermediates, glyceraldehyde-3-phosphate and dihydroxyacetonephosphate, and is mainly metabolized by glutathione-dependent glyoxalase 1 (Glo1) of the glyoxalase pathway (Rabbani et al., 2016b) (Figure 1A). The most susceptible targets in proteins to modification by ROS are cysteine residues which are oxidized to cystine and cysteine sulfenic and sulfonic acids (Winterbourn, 2008). The most susceptible targets in proteins to modification by MG are arginine residues which are glycated to hydroimidazolone MG$\mathrm{H} 1$ with loss of charge, all related electrostatic interactions and, typically, resistance to proteolytic cleavage close to the site of modification (Rabbani et al., 2016b) (Figure 1B).

Key to exploring if reactive metabolites of the host can be exploited to produce a virucidal response against SARS-CoV-2 is to identify proteomic vulnerabilities of the virus. Currently it is unknown if target amino acid residues of reactive metabolites are enriched in functional domains of the viral proteome, and if these targets amino acids are activated toward modification by reactive metabolites. It also unknown if there are investigational new drugs or current clinically approved drugs that increase reactive metabolites to virucidal levels in the cellular environment where SARS-CoV-2 undergo cell fusion and propagation. To address these gaps in knowledge, we initiated a series of studies using bioformatics tools, available proteomics data and a cell model used in SARS-CoV-2 virus propagation. Herein we predict the susceptibility of the SARS-CoV-2 virus to increased MG or "dicarbonyl stress" (Rabbani and Thornalley, 2015). This is based on enrichment of arginine residues in functional domains of the SARS-CoV-2 proteome and predicted activation of many of these arginine residues to modification by MG through neighboring group interactions. We also identified two clinical antitumor drugs that increase the cellular concentration of MG to virucidal levels and are candidates for consideration for repurposing for evaluation for treatment of COVID-19.

\section{MATERIALS AND METHODS}

\section{Reagents and Chemicals}

Doxorubicin, Paclitaxel, monoclonal anti-Glo1 antibody (rat), anti-Rat IgG (whole molecule)-Biotin conjugate, D-Lactic dehydrogenase were purchased from Sigma-Aldrich (Poole, Dorset, UK). Geneticin G-418 (potency rating - $700 \mu \mathrm{g}$ ) was purchased from Fisher Scientific (Loughborough, UK). S-pbromobenzylglutathione cyclopenyl diester (BBGD) was prepared an purifeid in-house, as described (Thornalley et al., 1996). The HEK293 cell line was purchased from the American Tissue Culture Collection (ATCC, Virginia, USA).

Plasmids, pIRES2-GLO1-EGFP and pIRES-EGFP, PJT laboratory were prepared and purified in-house, as described (Ahmed et al., 2008).

\section{Sequences of SARS CoV-2, SARS-CoV, and MERs and Human Host Proteins}

Reference sequences of the 29 proteins of the SARS-CoV-2 proteome (Table S1) and sequences of analogous proteins of SARS-CoV and Middle East Respiratory Syndrome (MERS) coronaviridae were obtained from the NBCI reference sequence database (www.ncbi.nlm.nih.gov). Sequences of reviewed proteins of the human proteome, 18,821 - excluding fetal proteins, were obtained from the UniProtKB database (www.uniprot.org).

\section{Receptor Binding Domain Analysis}

Receptor binding domain (RBD) analysis is a protein primary sequenced based informatics method to deduce amino acid residues in functional domains of proteins-defined as sites of protein-protein, protein-nucleic acid, and protein-ligand or substrate interaction. It is applicable to any protein. The optimized protocol uses a window of five amino acid residues moved sequentially along the sequence of a protein, assuming a gyration angle between two consecutive residues in the sequence of $100^{\circ}$, to deduce sequential mean Eisenberg hydrophobicity and mean dipole moment for the central amino acid. Values cannot be deduced for the two amino acids at the $\mathrm{N}$ - and $\mathrm{C}$ termini of proteins and they are therefore missing from the amino acid residue prevalence reports (Gallet et al., 2000). This approach had $80 \%$ accuracy when validated against a database of known interacting proteins (Gallet et al., 2000). We developed an $\mathrm{R}$ script to obtain mean hydrophobicity and hydrophobic moment for all UniProtKB proteins and SARS-CoV-2 proteins. 
A

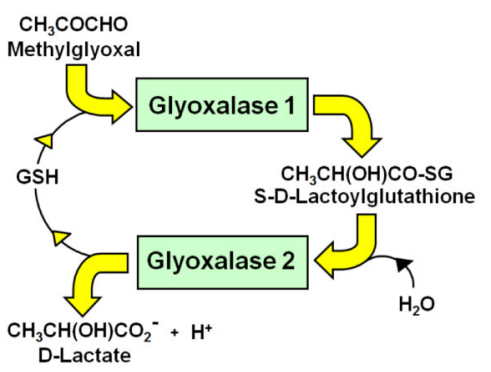

B $\quad$ co

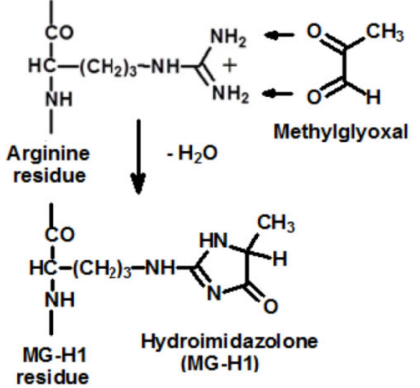

C

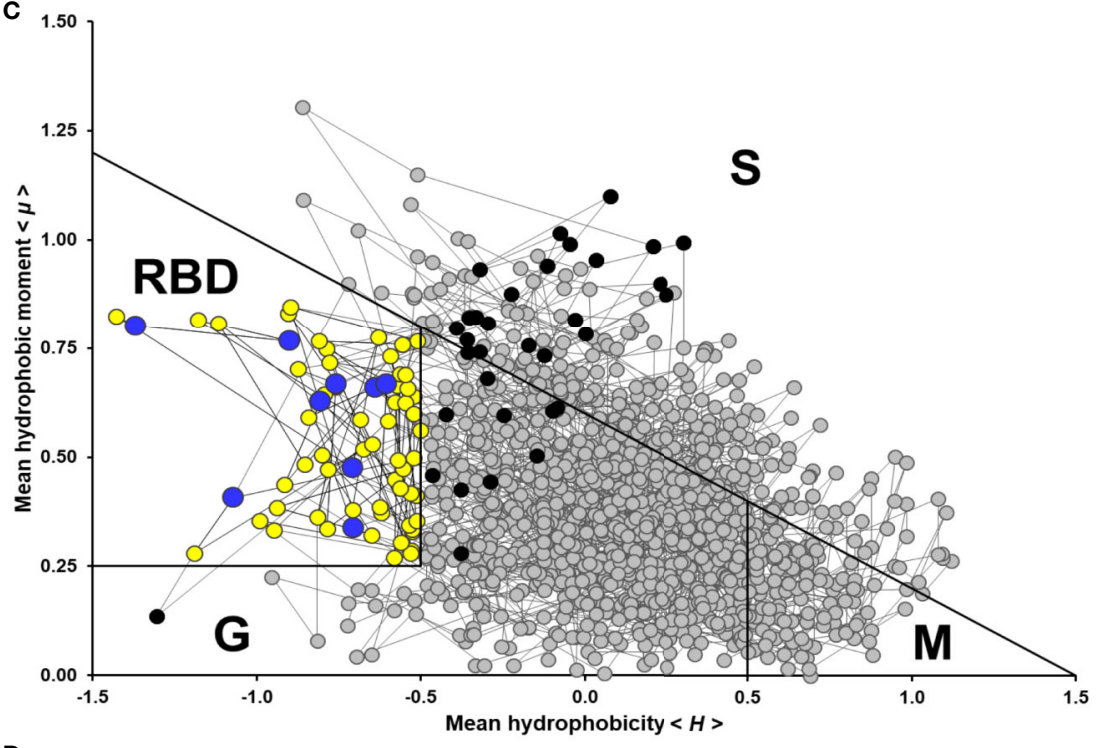

D

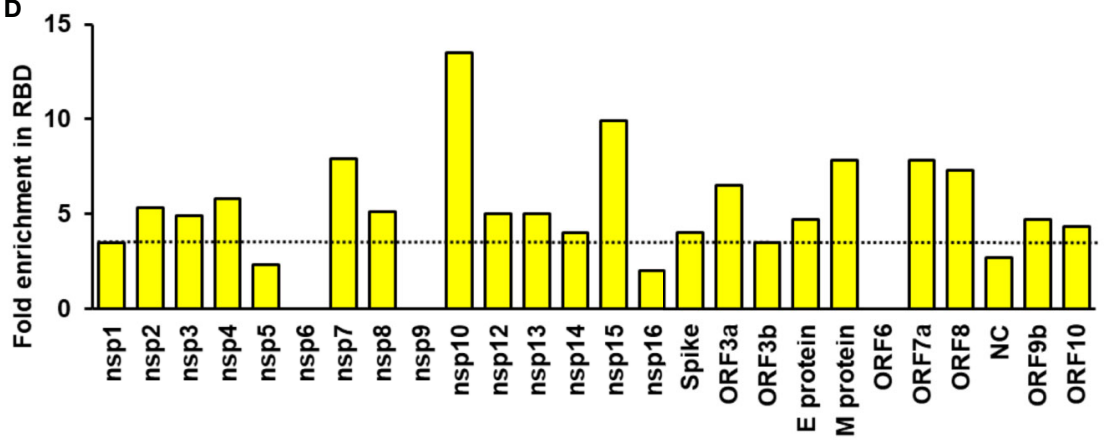

FIGURE 1 | Methylglyoxal-an endogenous arginine-modifying reactive metabolite and receptor binding domain analysis of functional arginines in the SARS-CoV-2 proteome. (A) Metabolism of methylglyoxal by the glyoxalase pathway (Rabbani et al., 2016b). (B) Modification of arginine residues by methylglyoxal (MG) to form hydroimidazolone, MG-H1. (C) Receptor binding domain (RBD) plot for SARS-CoV-2 Spike protein. Line-linked filled circles represent the primary sequence. The RBD is the area bound by the trapezium in the upper left-side region of the chart. Key: $\bigcirc$ arginine residue in the RBD; , arginine residues outside the RBD; O, other amino acid residues in the RBD; and $\bigcirc$, other amino acid residues outside the RBD. Other predicted domains: surface (S), globular (G), and membrane (M). (D) Arginine enrichment in individual proteins of SARS-CoV-2 proteins. Proteins not shown have no arginine residues (nsp11, ORF7b and ORF14). Dotted line mean fold enrichment of the human host proteome, 3.5.

\section{Prediction of Arginine Residues Activated for MG Modification}

Arginine residues of proteins which are activated toward reaction with $\mathrm{MG}$ by decrease of $\mathrm{pK}_{\mathrm{a}}$ of the guanidino side chain which facilitates formation of the MG-guanidino sidechain encounter (Rabbani and Thornalley, 2012; Rabbani et al., 2016a). Arginine residue sidechain $\mathrm{pK}_{\mathrm{a}}$ is decreased by interaction with neighboring amino acid residues with positively charged sidechains. For an $\alpha$ helix, interactions with lysine or arginine residues at positions -4 , $-3,+3$ and +4 in the sequence with respect to the target arginine residue are expected to decrease the arginine target residue $\mathrm{pK}_{\mathrm{a}}$ by side chain interaction along the side of the helix axis. Longer range 
interactions occur between these and other types of secondary structure domains in the tertiary structures of proteins where arginine residue $\mathrm{pK}_{\mathrm{a}}$ may be predicted from crystallographic data. We explored the peptide environments of arginine residues in predicted functional domains of the SARS-CoV-2 proteome, identifying arginine residues with neighboring interacting lysine and arginine residues, predicted secondary structure and predicted arginine residues target $\mathrm{pK}_{\mathrm{a}}$ where crystallographic data are available. Information on predicted secondary structure was extracted from in silico predicted models: nsp1, YxJyvF; nsp3, 5hYU6g; M-protein, 9LzAZz (http://3dbionotes.cnb.csic.es/ws/ covid19) (Waterhouse et al., 2018). Arginine side chain predicted $\mathrm{pK}_{\mathrm{a}}$ values are given, deduced by DelPhiPKa program (Wang et al., 2016) using nucleoprotein (NC) crystal structure (pdb file 6VYO; Chang et al., to be published) and AMBER forcefield (predictions were similar with CHARMM and PARSE forcefields). To identify similar arginines residues in SARS-CoV and MERS proteins, we used the Clustal Omega software on-line (Madeira et al., 2019).

\section{Culture of HEK293 Cells In Vitro}

The HEK293 cell line, seeding density $2 \times 10^{4}$ cells cm$^{-2}$, was cultured in Dulbecco's Modified Eagles Medium (DMEM) containing phenol red, L-glutamine and 4,500 mg/L glucose, supplemented with 10\% Fetal Bovine Serum (FBS), $100 \mathrm{U}$ penicillin and $0.1 \mathrm{mg} / \mathrm{ml}$ streptomycin. pIRES2-GLO1-EGFP plasmid (Glo1+ vector) and pIRES-EGFP plasmid (empty vector) were prepared as described (Ahmed et al., 2008). HEK293 cells were stably transfected with Glo1+ and empty vector using Lipofectamine 2000, according to the manufacturer's instructions (plasmid DNA: Lipofectamine $2000,1: 4)$. After $48 \mathrm{~h}$, cells were sub-cultured, G-418 disulphate was added ( $2 \mathrm{mg} / \mathrm{ml} ; 405 \mu \mathrm{g} / \mathrm{mg}$ potency) culture continued. Transfected colonies with GFP fluorescence were selected using a cloning disk $(3.2 \mathrm{~mm})$ and glass cylinder selector $(8 \mathrm{~mm}, 150 \mu \mathrm{l})$ and cultured further with G-418 disulphate $(1 \mathrm{mg} / \mathrm{ml}, 705 \mu \mathrm{g} / \mathrm{mg}$ potency) containing medium. Assessment of Glo1 activity and protein, as described (Arai et al., 2014; Xue et al., 2014), indicated a four- to fivefold increase in Glo1 activity and protein. HEK293 cells stably tranfected with empty and GLO1+ vectors were incubated with and without cell permeable Glo1 inhibitor, S-p-bromobenzylglutathione cyclopenyl diester (BBGD) (Thornalley et al., 1996), doxorubucin and paclitaxel at the concentrations indicated (diluted from $100 \mathrm{mM}$ stock solution in DMSO) for 2 days and effect on cell growth assessed by viable cell number counts, using the Trypan blue exclusion method and median growth inhibitory concentrations $\mathrm{GC}_{50}$ deduced. Cellular $\mathrm{MG}$ concentration and flux of formation of D-lactate, a surrogate measure of flux of formation of MG, was assayed as described (Rabbani and Thornalley, 2014; Irshad et al., 2019).

\section{Statistical Analysis}

Datasets were checked for normality of distribution and parametric statistical tests for assessment of significance of difference between study groups applied: Student's t-test for two groups and one-way ANOVA for 3 or more study groups.

\section{RESULTS}

\section{Enrichment of Arginine Residues in the Functional Domains in the SARS-CoV-2 Proteome}

We acquired primary amino acid sequences of the 29 proteins of the SARS-CoV-2 proteome (Table S1) and also, for comparison, 18,821 reviewed protein sequences of human host proteins from the UniProt Knowledgebase (UniProtKB; www.uniprot.org) excluding fetal proteins. We found a similar prevalence of cysteine and arginine residues in the viral proteome: $3.14 \%$ and $3.63 \%$, respectively (Table $\mathbf{1}$ ). We applied RBD analysis to identify functional domains of viral proteins and to thereby deduce the prevalence and enrichment of cysteine and arginine residues therein. The RBD analysis outcome is illustrated as a plot of mean hydrophobicity against mean dipole moment for the widow of 5 amino acid residues moved sequentially along the sequence of a protein. An example of the RBD analysis of the SARS-CoV-2 Spike protein is given in Figure 1C. Functional domains are located in a trapezium-shaped domain on the topleft side of the plot - regions of low mean hydrophobicity and high mean dipole moment of proximate groups of amino acid residues. This analysis showed that $4.8 \%$ of cysteine residues were in functional domains of the SARS-CoV-2 proteome whereas a much greater proportion of arginine residues, $30.7 \%$, were in functional domains. The enrichment of arginine residues in functional domains was 4.9-fold-the highest of any amino acid, whereas there was a slight negative enrichment, 0.8 -fold, or depletion of cysteine residues in functional domains. Other

TABLE 1 | Receptor binding domain of SARS-CoV-2 viral proteomes.

\begin{tabular}{|c|c|c|c|c|c|c|}
\hline \multirow[t]{2}{*}{ Amino acid } & \multicolumn{2}{|c|}{$\mathbf{N}$} & \multicolumn{2}{|c|}{ Prevalence } & \multirow[t]{2}{*}{ Proportion in RBD (\%) } & \multirow[t]{2}{*}{ Fold Enrichment } \\
\hline & All & RBD & All & RBD & & \\
\hline Arg & 358 & 110 & 3.63 & 17.7 & 30.7 & 4.9 \\
\hline Cys & 310 & 15 & 3.14 & 2.4 & 4.8 & 0.8 \\
\hline Met & 203 & 7 & 2.06 & 1.1 & 3.5 & 0.6 \\
\hline Tyr & 448 & 23 & 4.54 & 3.7 & 5.1 & 0.8 \\
\hline Trp & 113 & 2 & 1.15 & 0.3 & 1.8 & 0.3 \\
\hline
\end{tabular}

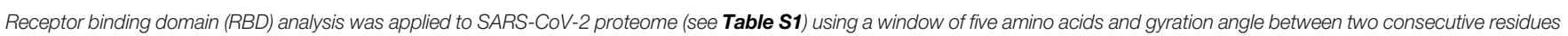
in the sequence of $100^{\circ}$ (Gallet et al., 2000). Complete amino acid profile is given in Table S2. 
amino acid residues susceptible to oxidative damage were also depleted in functional domains: met 0.6 , tyr 0.8 , and trp 0.3 (Table 1). The SARS-CoV-2 proteome is, therefore, resistant to oxidative inactivation but susceptible to functional inactivation by MG. For individual SARS-CoV-2 proteins, the majority had arginine residue enrichment in the functional domains greater than the mean of the human host proteome of $c a$. 3.5: range 2.313.5 (Figure 1D).

\section{Arginine Residues Activated for MG Modification by Predicted Neighboring Group Interaction}

We next sought to identify arginine residues in the predicted functional domains of SARS-CoV-2 proteins which are activated toward modification by MG based on potentially neighboring group side-chain interaction with arginine and lysine residues and, where crystallographic data are available, predicted target arginine residue $\mathrm{pK}_{\mathrm{a}}$. For example, in human serum albumin, neighboring group interactions with R186, R218 and R410 decrease the $\mathrm{pK}_{\mathrm{a}}$ values of their sidechain guanidino groups to $12.5,12.2$, and 12.5 from the basal $\mathrm{pK}_{\mathrm{a}}$ of 13.8 (Fitch et al., 2015). The reactivity with MG of these arginine residues increases by 20 - to 40 -fold through increase of the trace level conjugate base of the sidechain guanidino group (Fitch et al., 2015; Rabbani et al., 2016a). In low-level extent of modification of albumin by MG in experimental investigations in vitro and similar low-level extent of modification by MG of human serum albumin found similarly in vivo, MG was detected on these residues preferentially (Ahmed et al., 2005).

Applying RBD analysis and inspecting sequences for arginine or lysine residues at positions $-4,-3,+3$ and +4 with respect to the target arginine, we found the following number of arginine residues reactive toward $\mathrm{MG}$ modification and protein inactivation in predicted functional domains in SARS-CoV-2 proteins: nsp1, 2; nsp2, 3; nsp3, 3; nsp8, 1; nsp12, 1; nsp15, 2; spike protein, 2; M-protein, 1; NC, 10; and ORF10, 1. There were 25 functional arginines potentially activated for $\mathrm{MG}$ modification: 5 sites were in predicted $\alpha$-helices and 2 in NC with predicted $\mathrm{pK}_{\mathrm{a}}$ lowered by neighboring group interaction and thereby activated toward MG-modification (Table 2).

Uniquely for related coronaviridae, SARS-CoV-2 spike protein has an interacting arginine triad, $\mathrm{R}_{682} \mathrm{RAR}_{685}$, at the S1/S2 cleavage site (Andersen et al., 2020) with both R682 and R685 predicted sites susceptible to MG modification (Figure 2A). Modification of this triad by $\mathrm{MG}$ is expected to confer resistance to proteolytic cleavage by transmembrane serine proteases (TMPRSSs) and blocking cell fusion for virion entry into pulmonary alveolar epithelial and other cell target sites, uncoating and replication (Meng et al., 2020). Trapped in the extracellular environment, there is excepted to be an improved host immune response to the virus; $c f$. viral host immunity boosted by similar aldehyde-modifying agents (HerreraRodriguez et al., 2019).

SARS-CoV-2 nucleoprotein is highly susceptible to modification and functional inactivation by MG. Nucleoprotein binds the 3' end of the viral single strand RNA genome of SARSCoV-2 and it is arginine-rich, as is typical of RNA-binding proteins (Tan and Frankel, 1995). By analogy with SARS-CoV, residues $42-187$ are involved with RNA binding with R93 playing a critical role (Mcbride et al., 2014). It is predicted to have sites susceptible to MG modification. The crystal structure of a segment of nucleoprotein, residues $50-173$, is available and this enabled prediction of $\mathrm{pK}_{\mathrm{a}}$ values of arginine residues in this region. $\mathrm{pK}_{\mathrm{a}}$ shifts from 13.8 of inactivated arginine (Fitch et al.,

TABLE 2 | SARS-Cov-2, SARS-Cov, and MERS proteins with putative activated arginine residues in functional domains.

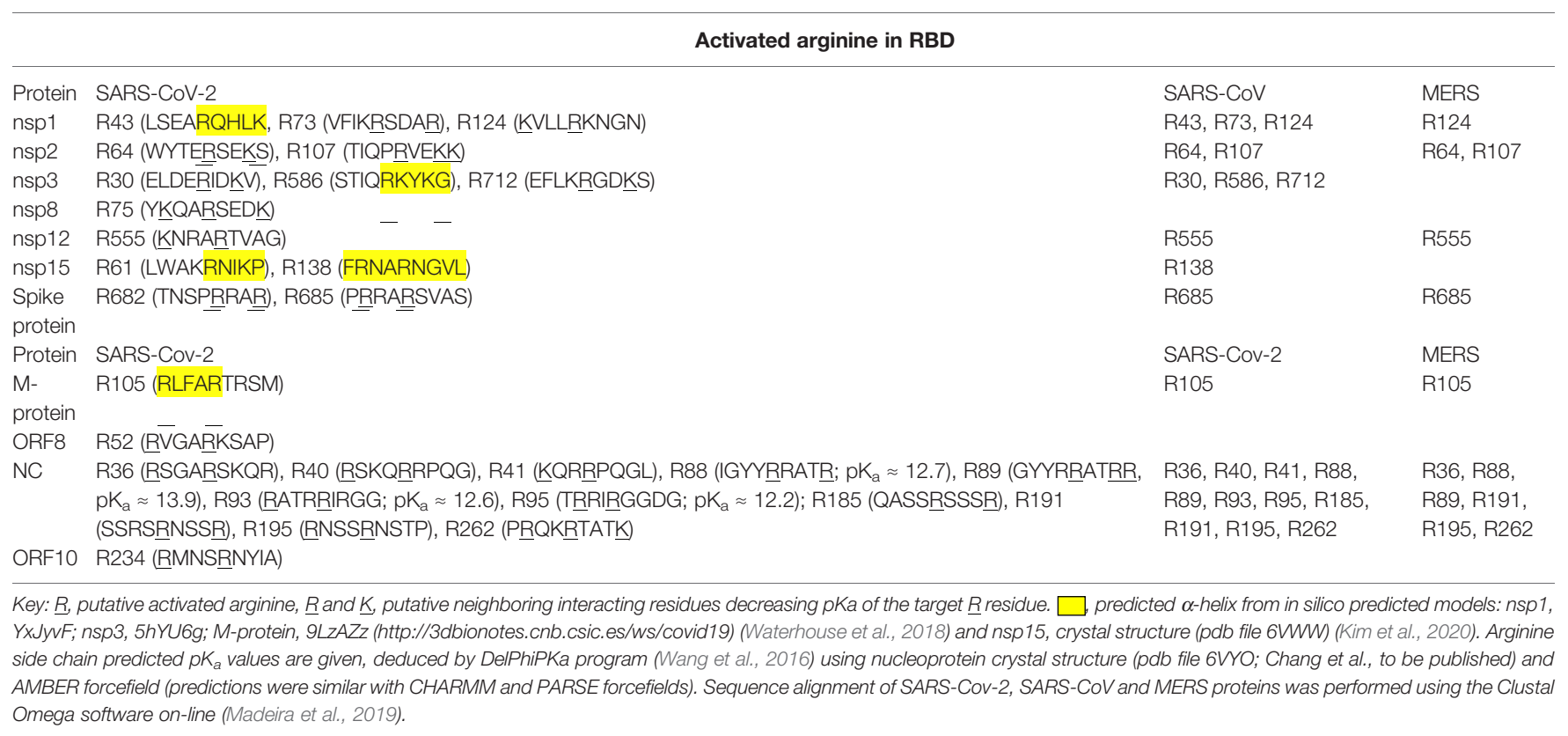




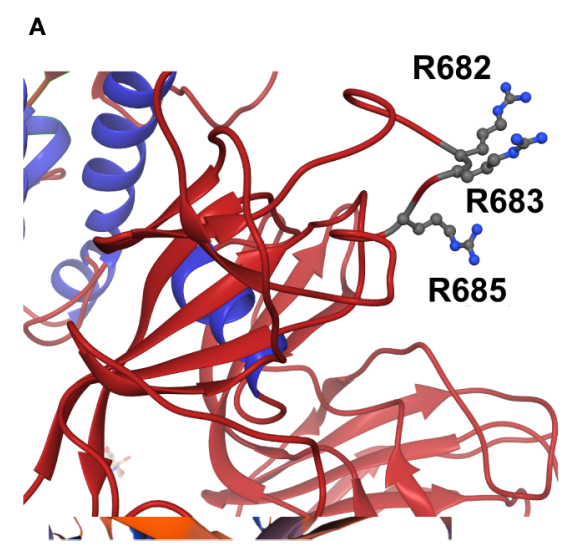

B

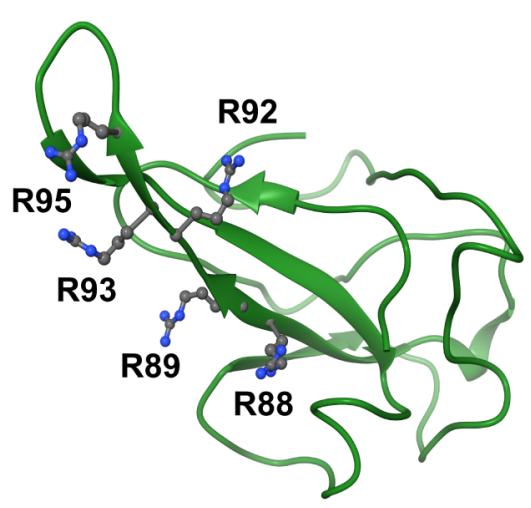

FIGURE 2 | Activation of functional arginine residues toward modification by methylglyoxal in the SARS-CoV-2 proteome. (A) Activated arginine residues triad of Spike protein, $R_{682} R_{683} A R_{685}$. (B) Activated arginine residue pentad of nucleoprotein, $R_{88} R_{89} A T R_{92} R_{93} I R_{95}$. Molecular graphics produced from Spike protein (pdb file 6VSB) (Wrapp et al., 2020) and nucleoprotein segment crystal structure (pdb file 6VYO; Chang et al., to be published) using Chimera 1.14 (Pettersen et al., 2004).

2015) for R93 and R95 indicated ca. 16 and 40-fold increased reactivity toward MG modification, compared to nonactivated target residue. These residues lie in a pentad of reactive arginine residues where MG modification at R93 and R95 is expected to inactive the nucleoprotein (Figure 2B). The SR-rich region of 182-196 is important for virus replication (Tylor et al., 2009) and is also a target for MG modification and inactivation at 3 sites: R185, R191 and R195. MG modification of the nucleoprotein, and also membrane protein, will block viral replication and virion assembly, respectively.

There are similar MG modification sites in functional domains of related coronaviridae. The proteome of severe acute respiratory syndrome coronavirus (SARS-CoV) had 23 similar MG modification sites to those of SARS-CoV-2, and MERS coronavirus proteome had 12 similar MG modification sites. In all of these coronaviridae there were multiple MG modification sites in functional domains of the nucleoprotein (Table 2). Given the high activation of multiple arginine residues in functional domains of the SARS-CoV-2 proteome, it is likely that pharmacological increase of endogenous MG concentration will produce modification at multiple susceptible and functional sites, producing protein inactivation and antiviral response.

\section{Pharmacological Increase of Cellular MG to Virucidal Levels by Cell Permeable Glyoxalase 1 Inhibitor and Clinical Antitumor Drugs, Doxorubicin, and Paclitaxel}

Antiviral activity of supraphysiological concentrations of MG was reported historically (De Bock et al., 1957). More recently, inhibition of cytopathic effect of strains of influenza B by MG was investigated. The most sensitive strain gave a median inhibitory concentration of $23 \pm 7 \mu \mathrm{M}$ MG (Charyasriwong et al., 2016). These studies assessed antiviral activity by cytopathic response - concentration of MG required to prevent $50 \%$ lysis of infected cells, using relatively high multiplicity of infection (MOI). Median effective concentrations for antiviral effects of pharmacological agents tend to be lower in physiologically relevant range of MOI than in pathogenic response assessment (Wang M. et al., 2020). These studies also used exogenous $M G$ in cellular in vitro models where $M G$ is rapidly metabolized by Glo1 and onward through the glyoxalase pathway to D-lactate (Rabbani et al., 2016b). The cellular concentration of $\mathrm{MG}$ is $1-4 \mu \mathrm{M}$ and the plasma concentration 130-250 nM (Rabbani and Thornalley, 2014; Xue et al., 2016; Irshad et al., 2019). The optimum approach to achieve an antiviral effect is to increase cellular MG concentration by inhibition of Glo1. BBGD is a potent cell permeable Glo1 inhibitor prodrug. It delivers the Glo1 competitive inhibitor, S-pbromobenzylglutathione $\left(\mathrm{K}_{\mathrm{i}}=160 \mathrm{nM}\right)$, into cells and has established antitumor and antimalarial activity (Thornalley et al., 1994; Thornalley et al., 1996) (Figure 3A). We studied the effect of BBGD and clinically approved antitumor drugs on the cellular concentration of MG in human HEK293 cells - a cellular model employed for SARS-CoV-2 propagation (Chien et al., 2016). BBGD increased the endogenous concentration of cellular MG by 4 -fold to $c a .20 \mu \mathrm{M}$ - a level similar to that which inhibited viral cytopathic activity (Charyasriwong et al., 2016).

We have a longstanding interest in anticancer activity of Glo1 inhibitors and overexpression of Glo1 in multidrug resistant tumors (Thornalley et al., 1996; Rabbani et al., 2018). Hence, we have been studying the likely involvement of increased MG in the mechanism of action of clinical antitumor drugs. Interestingly, we found clinical antitumor agents, doxorubicin and paclitaxel (Figure 3A), also increased cellular MG by a similar extent (Figure 3B). Increased $M G$ concentration induced by doxorubicin and paclitaxel is linked to increased glucose metabolism and related increased formation of $\mathrm{MG}$ as a byproduct of glycolysis. Indeed, flux of formation of D-lactate 
A

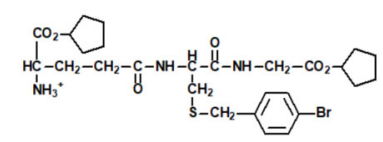

Glo1 inhibitor prodrug, BBGD

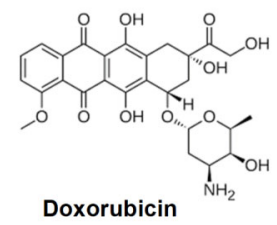

Doxorubicin

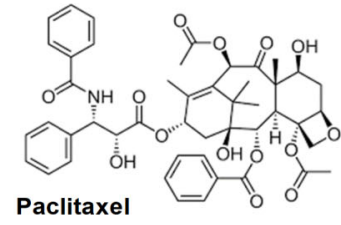

C

B
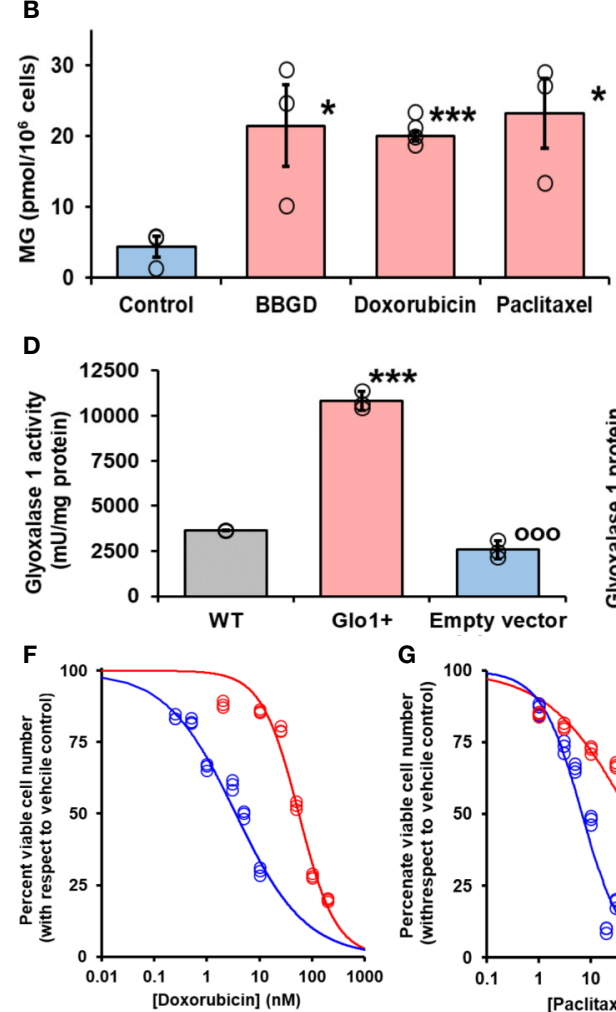

G

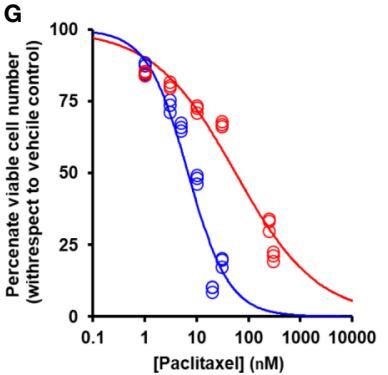

E Glo1 (21 kDa)

Control Doxorubicin Paclitaxel

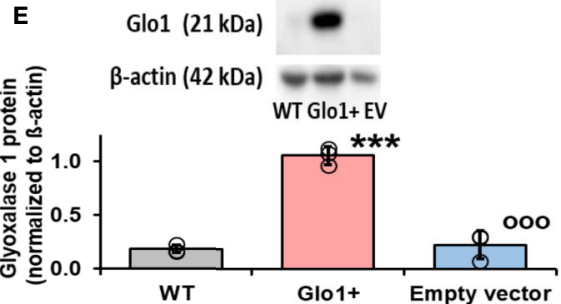

H

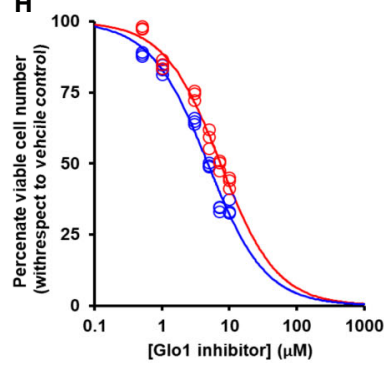

FIGURE 3 | Glyoxalase 1 inhibitor prodrug, doxorubicin and paclitaxel increase cellular concentration of methylglyoxal to virucidal levels. (A) Molecular structures of drugs. Glyoxalase 1 inhibitor prodrug, S-p-bromobenzylglutathione cyclopentyl diester (BBGD). Delivers competitive Glo1 inhibitor, S-p-bromobenzylglutathione, $\mathrm{K}_{\mathrm{i}}=$ 160 nM, into cells (Allen et al., 1993; Thornalley et al., 1996). Doxorubicin - topoisomerase inhibitor.(Tewey et al., 1984) Paclitaxel - stabilizer of microtubule

assembly (Schiff et al., 1979). (B, C) Increase in cellular methylglyoxal (MG) in HEK293 cells and flux of formation of D-lactate (surrogate for flux of MG), respectively, incubated in vitro with and without investigational agent and drugs indicated. For assay of MG, cells were incubated with and without treatment for $3 \mathrm{~h}$ and for flux of D-lactate incubated for $24 \mathrm{~h}$. Data are mean \pm SEM ( $n=3$ except $n=4$ for MG estimation with Doxorubicin; individual data points are shown). Drug concentrations: BBGD, $7.4 \mu \mathrm{M}$; doxorubicin, $6.0 \mathrm{nM}$, paclitaxel, $21 \mathrm{nM}$. Significance: b. $\mathrm{P}<0.02$ and c. $\mathrm{P}<0.01$ (one-way ANOVA) and * and ***, $\mathrm{P}<0.05$ and $\mathrm{P}<0.001$ with respect to control (t-test). (D, E) Activity and protein of Glo1, respectively, in HEK293 cells: wild-type (WT) and cells stably transfected to overexpress Glo1 (Glo1+) and empty vector (EV). Glo1 activity and protein were increased four- to fivefold. This was maintained for > 10 passages. (F-H) Effect of Glo1 expression on anti-proliferative activity. Key: blue - empty vector, red - Glo1 overexpression. HEK293 cells were incubated with and without treatment for 48 h. Data (six drug concentrations in triplicate) were fitted by nonlinear regression to the dose-response equation, $V=100 \times \mathrm{GC}_{50}{ }^{\mathrm{n}} /\left(\mathrm{GC}_{50}{ }^{\mathrm{n}}+\left[\right.\right.$ Drug $\left.^{\mathrm{n}}\right)$, solving for $\mathrm{GC}_{50}$ and, $\mathrm{n}$ (logistic regression coefficient). (F) Doxorubicin: empty vector, $\mathrm{GC}_{50}=3.54 \pm 0.28 \mathrm{nM}, \mathrm{n}=0.71 \pm 0.05$; Glo1+, $\mathrm{GC}_{50}=55.9 \pm 3.4 \mathrm{nM}, \mathrm{n}=1.24 \pm 0.10$ (16-fold resistance).

(G) Paclitaxel: empty vector, $\mathrm{GC}_{50}=6.8 \pm 1.0 \mathrm{nM}, \mathrm{n}=1.07 \pm 0.17$; and Glo1+, $\mathrm{GC}_{50}=56.4 \pm 7.2 \mathrm{nM}, \mathrm{n}=0.55 \pm 0.04$ (8-fold resistance). (H) BBGD: GC $50=$ $4.78 \pm 0.18 \mu \mathrm{M}, \mathrm{n}=1.02 \pm 0.05 ;$ and $\mathrm{Glo} 1+, \mathrm{GC}_{50}=7.37 \pm 0.30 \mu \mathrm{M}, \mathrm{n}=1.04 \pm 0.06$ (twofold resistance).

- a surrogate indicator of flux of formation of $\mathrm{MG}$-was increased by both drugs (Figure 3C).

Increase of cellular MG also likely contributes to the antiproliferative effect of BBGD (Rabbani et al., 2018). The involvement of $\mathrm{MG}$ in the antiproliferative activity of doxorubicin and paclitaxel is unknown. We explored this by determining the effect of overexpression of Glol on inhibition of
HEK293 cell growth. Vector-derived stable transfectant HEK293 cell lines were prepared with Glol expression increased four- to fivefold and empty vector transfectant control (Figures 3D, E), imposing a four- to fivefold increased rate of MG metabolism in Glo1 overexpressing cells. When these transfectant cell lines were treated with growth inhibitory concentrations of drugs, there was an increase of median growth inhibitory concentration $\mathrm{GC}_{50}$ 
value and resistance to inhibition of cell growth in HEK293 cells with stable overexpression of Glo1 (Figures $3 \mathbf{F}-\mathbf{H}$ ). The $\mathrm{GC}_{50}$ values were (mean $\pm \mathrm{SD}$; empty vector vs Glo1+): doxorubicin, $3.54 \pm 0.28 \mathrm{nM}$ vs $55.9 \pm 3.4 \mathrm{nM}$ (16-fold resistance); paclitaxel $6.8 \pm 1.0 \mathrm{nM}$ vs $56.4 \pm 7.2 \mathrm{nM}$ (eightfold resistance). For treatment with BBGD there was an antiproliferative effect with limited change in $\mathrm{GC}_{50}$ value with Glol overexpression: $4.78 \pm$ $0.18 \mu \mathrm{M}$ vs $7.37 \pm 0.30 \mu \mathrm{M}$ (2-fold resistance). The limited effect on antiproliferative effect of BBGD is expected as the delivered Glo1 inhibitor also inhibits the overexpression factor, Glo1.

\section{DISCUSSION}

The enrichment of arginine residues in functional domains of the SARS-CoV-2 proteome provides important evidence to support an arginine-modifying agent strategy for inactivation of the virus and virucidal activity. Arginine residues are also enriched in the human host proteome but less so that in SARS-CoV-2; 3.6 versus 4.9. This characteristic of the human proteome was noted previously (Gallet et al., 2000) —now updated herein with UniProtKB current sequence information. In addition, in the SARS-CoV-2 proteome there is a high number of arginine residues activated by neighboring groups for reaction with MG. This particularly applies to the nucleoprotein and, uniquely for the SARS-CoV-2 coronavirus, to the furin cleavage site of the spike protein. For the 25 arginine residues identified with predicted reactivity toward MG modification in the SARS-CoV-2 proteome, there is supporting secondary structure and predicted low $\mathrm{pK}_{\mathrm{a}}$ value evidence for seven of them. These arginine residue targets are in key proteins: nucleoprotein, M-protein, and Spike protein. A further important feature for susceptibility of viral proteins to $\mathrm{MG}$ modification is protein abundance: high abundance of a protein increases its susceptibility to reaction with MG. Previous earlier studies of the SARS virion suggested proteins of highest abundance were: nucleoprotein, M-protein, Spike protein and nsp3 (Neuman et al., 2008). Assuming a similar relative abundance of proteins in SARS-CoV-2, multiple arginine residues reactive toward modification and inactivation by MG were found in the 4 most abundant proteins of the SARS$\mathrm{CoV}-2$ proteome.

We predict the SARS-CoV-2 proteome is sensitive to modification by MG in functional sites. The proteome of human host alveolar cells is also likely to have increased modification by drug-induced increase of cellular MG. Protein domains sensitive to MG modification are chaperonin containing T-complex protein-1/T-ring complex protein-1 (CCT/TriC-1) chaperonins of protein (Irshad et al., 2019). Modification of these proteins is expected to be low but may delay folding of viral proteins and contribute to antiviral activity of drugs increasing the cellular concentration of MG.

We also explored use of proteomics data from previous studies where MG modification was detected at 411 in different sites in the cytosolic extract of human endothelial cells in culture (Irshad et al., 2019) in an attempt to identify a proteomic MG modification motif to map onto the SARS-CoV-2 proteome (data not shown). Although we could determine amino acid frequencies round the MG-modified arginine targets, there was a dropout of peptides (failure to detect) without lysine and arginine residues on the $\mathrm{N}$-terminal side of the arginine target in mass spectrometric detection. In comparisons with unmodified arginine target peptide sequences, this led to an artifactual enrichment of lysine and arginine residues on the $\mathrm{N}$ terminal side of the MG-modified arginine target. This peptide dropout was likely due to an additional missed cleavage by trypsin when the target arginines were modified by $M G$, making the related tryptic peptides difficult to detect in mass spectrometry analysis due to the loss of arginine residue charge and increased sequence length. This requires further investigation and likely studies with proteases other than trypsin in which MG modification changes tryptic peptide formation.

SARS-CoV-2 was rich with arginine residues in functional sites activated to MG modification. Other coronaviridae-SARSCoV-2 and MERS—had similar MG modification sites in protein crucial for virion viability - particularly the nucleoprotein. This suggests that pharmacological agents increasing cellular concentration of MG, inducing dicarbonyl stress, may have virucidal activity against multiple coronaviridae.

The SARS-CoV-2 proteome was predicted to be relatively resistant to oxidative damage because oxidant-sensitive cysteine residues were negatively enriched, or depleted, in functional sites; enrichment ratio 0.8 . A similarly depletion of methionine residues in functional sites was found, enrichment ratio 0.6 (Table 1), which are also susceptible to oxidative damage (Winterbourn, 2008). For induction of proteotoxicity, therefore, drugs which increase arginine-directed MG are predicted to be more effective than drugs which induce oxidative damage to proteins.

Doxorubicin and paclitaxel are clinical antiproliferative antitumor agents with mechanisms of action targeting inhibition of topoisomerase-II in DNA replication and stabilization of the interphase and microtubular network and mitotic spindle in mitosis, respectively (Schiff et al., 1979; Tewey et al., 1984). Herein, we show that increase in MG contributes to their mechanism of antiproliferative activity. Doxorubicin increases glucose metabolism by increasing expression of glucose transporter GLUT1 and hexokinase-2 (Demel et al., 2015). Paclitaxel stabilizes microtubules, decreasing free tubulin concentration; the latter increasing mitochondrial voltage-dependent anion channel (VDAC) activity and thereby in situ activity of hexokinase (Maldonado et al., 2010). These mechanisms are available in the lung epithelial cells primarily targeted by SARS-CoV-2 (Pezzulo et al., 2011; Lottes et al., 2014). Increased glucose metabolism produces a corresponding increase in the formation of MG - evidenced herein by increase in flux of formation of D-lactate; there may be disproportionately large increase in MG if expression of enzymes of onward metabolism of triosephosphates, triosephosphate isomerase and glyceraldehyde-3-phosphate dehydrogenase, are not increased along with hexokinase activity and glycolysis becomes 
dysregulated or unscheduled (Irshad et al., 2019; Rabbani and Thornalley, 2019).

BBGD has been evaluated previously in human cell cultures and tumor-bearing mice. It enters human cells in culture and hydrolyses to the Glo1 inhibitor, S-p-bromobenzyl-glutathione, and inhibits Glo1 with maximum cellular concentration of MG occurring after $3 \mathrm{~h}$ (Thornalley et al., 1996). In vivo studies were performed with BBGD and similar compounds in tumor bearing mice (Thornalley et al., 1996; Sakamoto et al., 2001). S-pBromobenzylglutathione is expected to eventually undergo excretion from cells and metabolism by the mercapturic acid pathway with urinary excretion of N-acetyl-S-pbromobenzylcysteine. Common strains of laboratory mice have markedly higher plasma esterase activity than human subjects, so an esterase-deficient strain of mouse is required in experimental investigations to avoid esterase-dependent inactivation of BBGD before reaching target tissues (Kavarana et al., 1999).

We envisage increased cellular MG interacting with the virus replication cycle as follows. SARS-coronaviruses replicate in the cytoplasm of infected host cells. Their replication complexes are associated with a reticulovesicular network of modified endoplasmic reticulum (ER) that integrates convoluted membranes and interconnected double membrane vesicles (Knoops et al., 2008). Viral RNA released by host cell fusion with the incoming virion is translated to express viral proteins. Multiple copies of the nucleoprotein enclose and package the genomic RNA. Spike protein, M- and E-proteins are inserted into the membrane of the rough ER and transported from the ER-toGolgi intermediate compartment to meet the nucleocapsid and assemble into particles by budding; M-protein playing a pivotal role, interacting with all viral assembly partners. Virions are transported through the constitutive secretory pathway out of the cell (De Haan and Rottier, 2005). Increasing cellular MG in virally-infected cells is expected to increase the modification of arginine residues of viral proteins - particularly nucleoprotein, spike protein and M-protein. Modification in functional sites of viral proteins, typically highly structured domains, converts cationic, hydrophilic arginine residues to uncharged hydrophobic MG-H1 residues. This produces protein misfolding, binding of misfolded proteins by heat shock proteins and ubiquitin ligases for degradation. Replication of SARS-CoV-2 is thereby slowed or terminated. Where viral proteins are modified by MG before folding, the change in hydrophobicity will likely impair correct folding and also direct the nascent polypeptide for ubiquitination and proteolysis. If some virions escape this proteotoxicity, MG modification on the spike protein may block or impair cell infectivity and thereby enhance viral immunogenicity; $c f$. $\beta$-propiolactone - an approach used in a SARS-CoV-2 vaccine in clinical evaluation (Gao et al., 2020). Indeed, further investigations may be merited to explore the use of MG modification to produce inactive virus for vaccine development studies. Most vaccines against SARS-CoV-2 in development contain whole or fragments of the spike protein (Jeyanathan et al., 2020).

Doxorubicin, paclitaxel and BBGD are expected to increase the cellular concentration of MG in human host tissues other than the lung. This may be advantageous as recent evidence suggests SARS-CoV-2 may directly infect endothelial cells of the kidney, heart and liver (Varga et al., 2020) and renal tubular epithelium and glomerular podocytes (Su et al., 2020). Increase of MG at these nonpulmonary sites may decrease viral load and decrease risk of vascular and renal complications of COVID-19. Relatively short-term treatment with drugs increasing cellular MG may be beneficial in patients with COVID-19. We acknowledge that, contrary to this, chronic increase of MG in clinical diabetes is rather associated with increased risk of vascular complications-including diabetic kidney disease (Rabbani et al., 2016b).

In the search for drugs to repurpose for COVID-19 disease, we suggest doxorubicin and paclitaxel be considered. These drugs have not been proposed hitherto although they have been evaluated for antiviral activity, particularly with respect to inhibition of viral helicase (Ash and Diekema, 1987; Bergamini et al., 1992; Borowski et al., 2002; Briguglio et al., 2011). Paclitaxel also suppressed inflammation in a murine model of bacterial pneumonia (Mirzapoiazova et al., 2007). However, a concern is the established adverse effects of these drugs found in cancer chemotherapy: bone marrow suppression (primarily neutropenia) and peripheral neuropathy for paclitaxel, and cumulative congestive heart failure for doxorubicin. Toxicity is related to dose and duration of treatment (Rowinsky, 1997; Barrett-Lee et al., 2009). Drug treatment of COVID-19 may be shorter than in cancer chemotherapy: for example, median hospitalization time of patients surviving severe symptoms of COVID-19 was 28 days (Wang L. et al., 2020) and a typical course of cancer chemotherapy with paclitaxel and doxorubicin is 6 months or longer (Rowinsky, 1997; Barrett-Lee et al., 2009). If high potency antiviral effect of these agents is found, low dose and short duration of treatment is expected to decrease risk of adverse effects.

The approach to drug repurposing for COVID-19 developed herein addresses the intrinsic vulnerability of SARS CoV-2 proteome to endogenous reactive metabolites, with respect to the human host, and identified drugs to exploit this. Other strategies for repurposing drugs are based on SARS CoV-2 protein interactions with human host proteins and drugs targeted to them, virion endosomal processing and viral protease inhibition (Chen et al., 2020; Gordon et al., 2020; Wang M. et al., 2020).

\section{CONCLUSIONS}

We provide evidence of vulnerability of SARS-CoV-2 to modification and inactivation by MG. We also reveal, for the first time, increase in cellular concentration of MG in the antiproliferative activity of doxorubicin and paclitaxel-thereby providing a mechanistic rationale for repurposing of these drugs against SARS-CoV-2 and treatment of COVID-19 disease. Doxorubicin and paclitaxel may have potential for application for treatment of COVID-19 and may now be considered for evaluation in SARS-CoV-2 live virus cultures and animal models. 


\section{DATA AVAILABILITY STATEMENT}

The raw data supporting the conclusions of this article will be made available by the authors, without undue reservation, to any qualified researcher.

\section{ETHICS STATEMENT}

All methods were carried out in accordance with relevant guidelines and regulations and all experimental protocols were approved by University of Warwick Genetic Modification \& Biosafety Committee (Project no. 305).

\section{AUTHOR CONTRIBUTIONS}

MA-M accessed protein sequence information on the SARSCoV-2, applied RBD analysis and produced the molecular graphics images. HA cultured HEK293 cells, prepared and propagated plasmids, prepared stable transfectant cell lines and performed metabolite and drug treatment studies. PW and AF collated and curated data on arginine sequence environments. MX provided technical guidance and support to HA and performed SARS-CoV2, SARS-CoV, and MERS sequence alignments. PJT assisted with MG analysis. NR and PJT acquired the funding, designed and supervised the studies, contributed to the data analysis and wrote the manuscript.

\section{REFERENCES}

Ahmed, N., Dobler, D., Dean, M., and Thornalley, P. J. (2005). Peptide mapping identifies hotspot site of modification in human serum albumin by methylglyoxal involved in ligand binding and esterase activity. J. Biol. Chem. 280, 5724-5732. doi: 10.1074/jbc.M410973200

Ahmed, U., Dobler, D., Larkin, S. J., Rabbani, N., and Thornalley, P. J. (2008). Reversal of hyperglycemia-induced angiogenesis deficit of human endothelial cells by overexpression of glyoxalase 1 in vitro. Ann. N. Y. Acad. Sci. 1126, 262264. doi: 10.1196/annals.1433.035

Allen, R. E., Lo, T. W. C., and Thornalley, P. J. (1993). A simplified method for the purification of human red blood cell glyoxalase I. Characteristics, immunoblotting and inhibitor studies. J. Prot. Chem. 12, 111-119. doi: $10.1007 / \mathrm{BF} 01026032$

Al-Motawa, M., Abbas, H., Wijten, P., De La Fuente, A., Xue, M., Rabbani, N., et al. (2020). Vulnerabilities of the SARS-CoV-2 virus to proteotoxicity opportunity for repurposed chemotherapy of COVID-19 infection. bioRxiv. 2020.2004.2007.029488. doi: 10.2139/ssrn.3582068

Andersen, K. G., Rambaut, A., Lipkin, W. I., Holmes, E. C., and Garry, R. F. (2020). The proximal origin of SARS-CoV-2. Nat. Med. 26, 450-452. doi: 10.1038/ s41591-020-0820-9

Arai, M., Nihonmatsu-Kikuchi, N., Itokawa, M., Rabbani, N., and Thornalley, P. J. (2014). Measurement of glyoxalase activities. Biochem. Soc. Trans. 42, 491-494. doi: 10.1042/BST20140010

Ash, R. J., and Diekema, K. A. (1987). Inhibition of herpes simplex virus replication by anthracycline compounds. Antiviral Res. 8, 71-83. doi: 10.1016/0166-3542(87)90078-7

Barrett-Lee, P. J., Dixon, J. M., Farrell, C., Jones, A., Leonard, R., Murray, N., et al. (2009). Expert opinion on the use of anthracyclines in patients with advanced breast cancer at cardiac risk. Ann. Oncol. 20, 816-827. doi: 10.1093/annonc/ $\operatorname{mdn} 728$
All authors contributed to the article and approved the submitted version.

\section{FUNDING}

MA-M thanks the Qatar Foundation for a PhD studentship. PJT thanks the Qatar Foundation for funding his research program (project code QB-14). NR thanks Qatar University for funding her COVID-19 research (project code QU ERGCMED-2020-1).

\section{ACKNOWLEDGMENTS}

This manuscript has been released as a pre-print at BioRxiv (AlMotawa et al., 2020).

\section{SUPPLEMENTARY MATERIAL}

The Supplementary Material for this article can be found online at: https://www.frontiersin.org/articles/10.3389/fphar.2020.585408/ full\#supplementary-material

SUPPLEMENTARY TABLE 1 | SARS-CoV-2 viral proteome. *Sequence from reference (Chan et al., 2020).

SUPPLEMENTARY TABLE 2 | Receptor binding domain of SARS-CoV-2 viral proteome. RBD analysis was applied to SARS-CoV-2 proteome (see Table S1) using a window of 5 amino acids and gyration angle between two consecutive residues in the sequence of $100^{\circ}$ (Gallet et al., 2000).

Bergamini, A., Perno, C. F., Balzarini, J., Capozzi, M., Marinelli, L., Milanese, G. et al. (1992). Selective Inhibition of HIV Replication by Adriamycin in Macrophages But Not in Lymphocytes. AIDS Res. Hum. Retroviruses 8, 1239-1247. doi: 10.1089/aid.1992.8.1239

Borowski, P., Schalinski, S., and Schmitz, H. (2002). Nucleotide triphosphatase/ helicase of hepatitis $\mathrm{C}$ virus as a target for antiviral therapy. Antiviral Res. 55, 397-412. doi: 10.1016/S0166-3542(02)00096-7

Briguglio, I., Piras, S., Corona, P., and Carta, A. (2011). Inhibition of RNA Helicases of ssRNA(+) Virus Belonging to Flaviviridae, Coronaviridae and Picornaviridae Families. Int. J. Med. Chem. 2011, 213135. doi: 10.1155/2011/ 213135

Chan, J. F.-W., Kok, K.-H., Zhu, Z., Chu, H., To, K. K.-W., Yuan, S., et al. (2020). Genomic characterization of the 2019 novel human-pathogenic coronavirus isolated from a patient with atypical pneumonia after visiting Wuhan. Emerg. Microbes Infect. 9, 221-236. doi: 10.1080/22221751.2020.1719902

Charyasriwong, S., Haruyama, T., and Kobayashi, N. (2016). In vitro evaluation of the antiviral activity of methylglyoxal against influenza B virus infection. Drug Discover. Ther. 10, 201-210. doi: 10.5582/ddt.2016.01045

Chen, Y., Yiu, C., and Wong, K. (2020). Prediction of the SARS-CoV-2 (2019$\mathrm{nCoV}$ ) 3C-like protease (3CLpro) structure: virtual screening reveals velpatasvir, ledipasvir, and other drug repurposing candidates [version 1; peer review: 3 approved]. F1000Res. 9, 129 (1-9). doi: 10.12688/ f1000research.22457.2

Chien, H.-C., Zur, A. A., Maurer, T. S., Yee, S. W., Tolsma, J., Jasper, P., et al. (2016). Rapid Method To Determine Intracellular Drug Concentrations in Cellular Uptake Assays: Application to Metformin in Organic Cation Transporter 1-Transfected Human Embryonic Kidney 293 Cells. Drug Metab. Disposition: Biol. Fate Chem. 44, 356-364. doi: 10.1124/ dmd.115.066647

De Bock, C. A., Brug, J., and Walop, J. N. (1957). Antiviral Activity of Glyoxals. Nature 179, 706-707. doi: 10.1038/179706a0 
De Haan, C., and Rottier, P. J. M. (2005). Molecular interactions in the assembly of coronaviruses. Adv. Virus Res. 64, 165-230. doi: 10.1016/S0065-3527(05) 64006-7

Demel, H. R., Feuerecker, B., Piontek, G., Seidl, C., Blechert, B., Pickhard, A., et al. (2015). Effects of topoisomerase inhibitors that induce DNA damage response on glucose metabolism and PI3K/Akt/mTOR signaling in multiple myeloma cells. Am. J. Cancer Res. 5, 1649-1664.

Fitch, C. A., Platzer, G., Okon, M., Garcia-Moreno, B. E., and Mcintosh, L. P. (2015). Arginine: Its pKa value revisited. Protein Sci: Publ. Protein Soc. 24, 752-761. doi: 10.1002/pro.2647

Gallet, X., Charloteaux, B., Thomas, A., and Braseur, R. (2000). A fast method to predict protein interaction sites from sequences. J. Mol. Biol. 302, 917-926. doi: 10.1006/jmbi.2000.4092

Gao, Q., Bao, L., Mao, H., Wang, L., Xu, K., Yang, M., et al. (2020). Development of an inactivated vaccine candidate for SARS-CoV-2. Science 369, 77-81. doi: 10.1126/science.abc1932

Gordon, D. E., Jang, G. M., Bouhaddou, M., Xu, J., Obernier, K., O’meara, M. J., et al. (2020). A SARS-CoV-2-Human Protein-Protein Interaction Map Reveals Drug Targets and Potential Drug-Repurposing. bioRxiv. 583, 459-468. doi: 10.1101/2020.03.22.002386

Herrera-Rodriguez, J., Signorazzi, A., Holtrop, M., De Vries-Idema, J., and Huckriede, A. (2019). Inactivated or damaged? Comparing the effect of inactivation methods on influenza virions to optimize vaccine production. Vaccine 37, 1630-1637. doi: 10.1016/j.vaccine.2019.01.086

Irshad, Z., Xue, M., Ashour, A., Larkin, J. R., Thornalley, P. J., and Rabbani, N. (2019). Activation of the unfolded protein response in high glucose treated endothelial cells is mediated by methylglyoxal. Sci. Rep. 9, 7889. doi: 10.1038/ s41598-019-44358-1

Jeyanathan, M., Afkhami, S., Smaill, F., Miller, M. S., Lichty, B. D., and Xing, Z. (2020). Immunological considerations for COVID-19 vaccine strategies. Nat Rev Immunol. 20, 615-632. doi: 10.1038/s41577-020-00434-6

Kavarana, M. J., Kovaleva, E. G., Creighton, D. J., Wollman, M. B., and Eiseman, J. L. (1999). Mechanism-Based Competitive Inhibitors of Glyoxalase I: Intracellular Delivery, in Vitro Antitumor Activities, and Stabilities in Human Serum and Mouse Serum. J. Med. Chem. 42, 221-228. doi: 10.1021/jm9708036

Kim, Y., Jedrzejczak, R., Maltseva, N. I., Endres, M., Godzik, A., Michalska, K., et al. (2020). Crystal structure of Nsp15 endoribonuclease NendoU from SARS-CoV-2. Protein Sci. 29, 1596-1605. doi: 10.1101/2020.03.02.968388

Knoops, K., Kikkert, M., Worm, S.H.E.V.D., Zevenhoven-Dobbe, J. C., Van Der Meer, Y., Koster, A. J., et al. (2008). SARS-Coronavirus Replication Is Supported by a Reticulovesicular Network of Modified Endoplasmic Reticulum. PloS Biol. 6, e226. doi: 10.1371/journal.pbio.0060226

Lottes, R. G., Newton, D. A., Spyropoulos, D. D., and Baatz, J. E. (2014). Alveolar type II cells maintain bioenergetic homeostasis in hypoxia through metabolic and molecular adaptation. Am. J. Physiol. Lung Cell. Mol. Physiol. 306, L947L955. doi: 10.1152/ajplung.00298.2013

Madeira, F., Park, Y. M., Lee, J., Buso, N., Gur, T., Madhusoodanan, N., et al. (2019). The EMBL-EBI search and sequence analysis tools APIs in 2019. Nucleic Acids Res. 47, W636-W641. doi: 10.1093/nar/gkz268

Maldonado, E. N., Patnaik, J., Mullins, M. R., and Lemasters, J. J. (2010). Free tubulin modulates mitochondrial membrane potential in cancer cells. Cancer Res. 70, 10192-10201. doi: 10.1158/0008-5472.CAN-10-2429

Mcbride, R., Van Zyl, M., and Fielding, B. C. (2014). The Coronavirus Nucleocapsid Is a Multifunctional Protein. Viruses 6, 2991-3018. doi: 10.3390/ v6082991

Meng, T., Cao, H., Zhang, H., Kang, Z., Xu, D., Gong, H., et al. (2020). The insert sequence in SARS-CoV-2 enhances spike protein cleavage by TMPRSS. bioRxiv. 2020.2002.2008.926006. doi: 10.1101/2020.02.08.926006

Mirzapoiazova, T., Kolosova, I. A., Moreno, L., Sammani, S., Garcia, J. G., and Verin, A. D. (2007). Suppression of endotoxin-induced inflammation by taxol. Eur. Respir. J. 30, 429-435. doi: 10.1183/09031936.00154206

Murphy, M. P., Holmgren, A., Larsson, N. G., Halliwell, B., Chang, C. J., Kalyanaraman, B., et al. (2011). Unraveling the Biological Roles of Reactive Oxygen Species. Cell Metab. 13, 361-366. doi: 10.1016/j.cmet.2011.03.010

Neuman, B. W., Joseph, J. S., Saikatendu, K. S., Serrano, P., Chatterjee, A., Johnson, M. A., et al. (2008). Proteomics Analysis Unravels the Functional Repertoire of Coronavirus Nonstructural Protein 3. J. Virol. 82, 5279-5294. doi: 10.1128/ JVI.02631-07
Pettersen, E. F., Goddard, T. D., Huang, C. C., Couch, G. S., Greenblatt, D. M., Meng, E. C., et al. (2004). UCSF Chimera-a visualization system for exploratory research and analysis. J. Comput. Chem. 25, 1605-1612. doi: 10.1002/jcc.20084

Pezzulo, A. A., Gutiérrez, J., Duschner, K. S., Mcconnell, K. S., Taft, P. J., Ernst, S. E., et al. (2011). Glucose Depletion in the Airway Surface Liquid Is Essential for Sterility of the Airways. PloS One 6, e16166. doi: 10.1371/journal.pone.0016166

Rabbani, N., and Thornalley, P. J. (2012). Methylglyoxal, glyoxalase 1 and the dicarbonyl proteome. Amino Acids 42, 1133-1142. doi: 10.1007/s00726-0100783-0

Rabbani, N., and Thornalley, P. J. (2014). Measurement of methylglyoxal by stable isotopic dilution analysis LC-MS/MS with corroborative prediction in physiological samples. Nat. Protoc. 9, 1969-1979. doi: 10.1038/nprot.2014.129

Rabbani, N., and Thornalley, P. J. (2015). Dicarbonyl stress in cell and tissue dysfunction contributing to ageing and disease. Biochem. Biophys. Res. Commun. 458, 221-226. doi: 10.1016/j.bbrc.2015.01.140

Rabbani, N., and Thornalley, P. J. (2019). Hexokinase-2 Glycolytic Overload in Diabetes and Ischemia-Reperfusion Injury. Trends Endocrinol. Metab. 30, 419-431. doi: 10.1016/j.tem.2019.04.011

Rabbani, N., Ashour, A., and Thornalley, P. J. (2016a). Mass spectrometric determination of early and advanced glycation in biology. Glycoconjugate J. 33, 553-568. doi: 10.1007/s10719-016-9709-8

Rabbani, N., Xue, M., and Thornalley, P. J. (2016b). Methylglyoxal-induced dicarbonyl stress in aging and disease: first steps towards glyoxalase 1-based treatments. Clin. Sci. 130, 1677-1696. doi: 10.1042/CS20160025

Rabbani, N., Xue, M., Weickert, M. O., and Thornalley, P. J. (2018). Multiple roles of glyoxalase 1-mediated suppression of methylglyoxal glycation in cancer biology-Involvement in tumour suppression, tumour growth, multidrug resistance and target for chemotherapy. Semin. Cancer Biol. 49, 83-93. doi: 10.1016/j.semcancer.2017.05.006

Rowinsky, E. K. (1997). The development and clinical utility of the taxane class of antimicrotubule chemotherapy agents. Annu. Rev. Med. 48, 353-374. doi: 10.1146/annurev.med.48.1.353

Sakamoto, H., Mashima, T., Sato, S., Hashimoto, Y., Yamori, T., and Tsuruo, T. (2001). Selective activation of apoptosis program by S-p-bromobenzylglutathione cyclopentyl diester in glyoxalase I-overexpressing human lung cancer cells. Clin. Cancer Res. 7, 2513-2518.

Schiff, P. B., Fant, J., and Horwitz, S. B. (1979). Promotion of microtubule assembly in vitro by taxol. Nature 277, 665-667. doi: 10.1038/277665a0

Su, H., Yang, M., Wan, C., Yi, L.-X., Tang, F., Zhu, H.-Y., et al. (2020). Renal histopathological analysis of 26 postmortem findings of patients with COVID19 in China. Kidney Int. 98, 219-227. doi: 10.1016/j.kint.2020.04.003

Tan, R., and Frankel, A. D. (1995). Structural variety of arginine-rich RNAbinding peptides. Proc. Natl. Acad. Sci. U. States America 92, 5282-5286. doi: 10.1073/pnas.92.12.5282

Tewey, K., Rowe, T., Yang, L., Halligan, B., and Liu, L. (1984). Adriamycininduced DNA damage mediated by mammalian DNA topoisomerase II. Science 226, 466-468. doi: 10.1126/science.6093249

Thornalley, P. J., Strath, M., and Wilson, R. J. M. (1994). Anti-malarial activity in vitro of the glyoxalase I inhibitor diester, S-p-bromobenzylglutathione diethyl ester. Biochem. Pharmacol. 268, 14189-14825. doi: 10.1016/0006-2952(94) 90035-3

Thornalley, P. J., Edwards, L. G., Kang, Y., Wyatt, C., Davies, N., Ladan, M. J., et al. (1996). Antitumour activity of S-p- bromobenzylglutathione cyclopentyl diester in vitro and in vivo. Inhibition of glyoxalase I and induction of apoptosis. Biochem. Pharmacol. 51, 1365-1372. doi: 10.1016/0006-2952(96) 00059-7

Tylor, S., Andonov, A., Cutts, T., Cao, J., Grudesky, E., Van Domselaar, G., et al. (2009). The SR-rich motif in SARS-CoV nucleocapsid protein is important for virus replication. Can. J. Microbiol. 55, 254-260. doi: 10.1139/W08-139

Varga, Z., Flammer, A. J., Steiger, P., Haberecker, M., Andermatt, R., Zinkernagel, A. S., et al. (2020). Endothelial cell infection and endotheliitis in COVID-19. Lancet 395, 1417-148. doi: 10.1016/S0140-6736(20)30937-5

Wang, L., Zhang, M., and Alexov, E. (2016). DelPhiPKa web server: predicting pKa of proteins, RNAs and DNAs. Bioinformatics 32, 614-615. doi: 10.1093/ bioinformatics/btv607

Wang, L., He, W., Yu, X., Hu, D., Bao, M., Liu, H., et al. (2020). Coronavirus disease 2019 in elderly patients: Characteristics and prognostic factors based on 4-week follow-up. J. Infect. 80, 639-645. doi: 10.1016/j.jinf.2020.03.019 
Wang, M., Cao, R., Zhang, L., Yang, X., Liu, J., Xu, M., et al. (2020). Remdesivir and chloroquine effectively inhibit the recently emerged novel coronavirus, (2019-nCoV) in vitro. Cell Res. 30, 269-271. doi: 10.1038/s41422-0200282-0

Waterhouse, A., Bertoni, M., Bienert, S., Studer, G., Tauriello, G., Gumienny, R., et al. (2018). SWISS-MODEL: homology modelling of protein structures and complexes. Nucleic Acids Res. 46, W296-W303. doi: 10.1093/nar/gky427

Winterbourn, C. C. (2008). Reconciling the chemistry and biology of reactive oxygen species. Nat. Chem. Biol. 4, 278-286. doi: 10.1038/ nchembio. 85

Wrapp, D., Wang, N., Corbett, K. S., Goldsmith, J. A., Hsieh, C.-L., Abiona, O., et al. (2020). Cryo-EM structure of the 2019-nCoV spike in the prefusion conformation. Science 367, 1260-1263. doi: 10.1126/science.abb2507

Xue, M., Rabbani, N., and Thornalley, P. J. (2014). Measurement of glyoxalase gene expression. Biochem. Soc. Trans. 42, 495-499. doi: 10.1042/ BST20140026
Xue, M., Weickert, M. O., Qureshi, S., Ngianga-Bakwin, K., Anwar, A., Waldron, M., et al. (2016). Improved glycemic control and vascular function in overweight and obese subjects by glyoxalase 1 inducer formulation. Diabetes $65,2282-2294$. doi: $10.2337 / \mathrm{db} 16-0153$

Conflict of Interest: The authors declare that the research was conducted in the absence of any commercial or financial relationships that could be construed as a potential conflict of interest.

Copyright (C) 2020 Al-Motawa, Abbas, Wijten, de la Fuente, Xue, Rabbani and Thornalley. This is an open-access article distributed under the terms of the Creative Commons Attribution License (CC BY). The use, distribution or reproduction in other forums is permitted, provided the original author(s) and the copyright owner(s) are credited and that the original publication in this journal is cited, in accordance with accepted academic practice. No use, distribution or reproduction is permitted which does not comply with these terms. 